gr-qc/9510038/ DESY 95-190

\title{
Quantum Field Theory at Finite Temperature and Cosmological Perturbations*
}

\author{
Anton K. Rebhan ${ }^{\dagger}$ \\ DESY, Gruppe Theorie, \\ Notkestraße 85, D-22603 Hamburg, Germany
}

\begin{abstract}
It is shown how quantum field theory at finite temperature can be used to set up self-consistent and gauge invariant equations for cosmological perturbations sustained by an ultrarelativistic plasma. While in the collisionless case, the results are equivalent to those obtained from the Einstein-Vlasov equations, weak self-interactions in the plasma turn out to require the full machinery of perturbative thermal field theories such as resummation of hard thermal loops. Nevertheless it is still possible to use the same methods that yielded exact solutions in the collisionless case.
\end{abstract}

In order to account for the present large-scale structure of the universe such as galaxies, clusters, superclusters, voids, etc., [1] a cosmological model built on homogeneous and isotropic geometries requires certain imperfections in its symmetries. Through the universally attractive nature of gravitations, initially small perturbations can grow, in particular once that the universe becomes matter dominated and the (maximal) pressure provided by radiation has become inoperative. This picture, which is based on a big-bang scenario, has found dramatic support by the search for and discovery of tiny anisotropies in the cosmic micro-wave background, which in a Friedmann-Robertson-Walker (FRW) universe have wavelengths far exceeding the size of the Hubble horizon at the time when this radiation decoupled from the primordial matter [2].

Whatever the origin of these small deviations from homogeneity and isotropy, there is a rather long epoch of radiation domination which is thought to be well described by a nearly perfect FRW model with metric perturbations evolving in a linear regime. The basic equations for these "cosmological perturbations" are nothing else than the perturbed Einstein equations,

\footnotetext{
*Talk presented at the Third Workshop on Quantum Field Theory under the Influence of External Conditions, Leipzig, Germany, 18-22 September, 1995

$\dagger$ On leave of absence from Institut für Theoretische Physik der Technischen Universität Wien, Wiedner Hauptstr. 8-10, A-1040 Wien, Austria; email: rebhana@email.tuwien.ac.at
} 


$$
\delta G^{\mu \nu} \equiv \frac{\delta\left(R^{\mu \nu}-\frac{1}{2} g^{\mu \nu} R\right)}{\delta g^{\alpha \beta}} \delta g^{\alpha \beta}=-8 \pi G \delta T^{\mu \nu} .
$$

In order to have a close set of equations, these have to be supplied with information on the response $\delta T^{\mu \nu}$ of the energy-momentum tensor to metric perturbations $\delta g^{\alpha \beta}$. In a hydrodynamic approach, this is done by sufficiently restricting the form of $\delta T^{\mu \nu}$, specifying the equations of state, and imposing covariant conversation of the full energy-momentum tensor in the perturbed geometry. The simplest case is the one of a perfect (radiation) fluid, which has been studied in the pioneering work of Lifshitz [3]. Many generalizations have since been worked out, and have been cast into a gauge invariant form by Bardeen [4]. A modern geometrical justification and generalization has been given recently by Ellis and co-authors [5].

A more fundamental description of the behaviour of the primordial matter, which in the early universe is mostly a hot plasma of elementary particles, is usually implemented through kinetic theory [6]. However, a truly fundamental description eventually has to take into account quantum field theory. In the following I shall show that an interesting part of the theory of cosmological perturbations can be investigated through the techniques developed for quantum field theory at finite temperature [7], namely the case of a weakly interacting ultrarelativistic particle plasma. In the limiting case of a collisionless ultrarelativistic plasma it turns out to be even possible to obtain exact analytic results [8] where only numerical ones where known before; in the case of weak self-interactions one can still find analytic results [9] which involve such issues as resummation of hard thermal loops that would be very difficult to include in a (quantum) kinetic approach.

For temperatures $T \ll m_{\text {Planck }}$ it is sufficient to treat the gravitational field as a classical background field. The energy-momentum tensor can then be defined by the one-point function

$$
T_{\mu \nu}(x)=\frac{2}{\sqrt{-g}} \frac{\delta \Gamma[g]}{\delta g^{\mu \nu}},
$$

where $\Gamma[g]$ is the effective action functional that contains all the contributions besides the classical Einstein-Hilbert action. When derived from this effective action, covariant conservation of the energy-momentum tensor is automatic and need not be imposed as a constraint.

The response under perturbations in the metric field is given by

$$
\delta T_{\mu \nu}(x)=\int d^{4} y \frac{\delta T_{\mu \nu}(x)}{\delta g^{\alpha \beta}(y)} \delta g^{\alpha \beta}(y) .
$$

Hence, $\delta T_{\mu \nu}$ is determined by the gravitational polarization tensor (or "thermal graviton self-energy")

$$
\Pi_{\mu \nu \alpha \beta}(x, y) \equiv \frac{\delta^{2} \Gamma}{\delta g^{\mu \nu}(x) \delta g^{\alpha \beta}(y)}=\frac{1}{2} \frac{\delta\left(\sqrt{-g} T_{\mu \nu}(x)\right)}{\delta g^{\alpha \beta}(y)} .
$$

In particle physics terminology, (2) and (41) are the sets of one-particle irreducible diagrams with one and two external graviton line(s) in the background field $g_{\mu \nu}$ given by the cosmological model on which one wants to study the dynamics of cosmological perturbations. The concept of thermal equilibrium makes rigorous sense in conformally trivial situations 
where $g_{\mu \nu}(x)=\sigma(x) \eta_{\mu \nu}$. This is indeed the case with almost all of the cosmological models of interest. If one knows how the effective action transforms under conformal rescalings of the metric, then the entire problem of determining the highly nonlocal function (四) (and thus the response of the plasma) can be reduced to the evaluation of Feynman diagrams in flat space, where momentum-space techniques can be used. In flat space, temperature can be introduced through periodicity in imaginary time, and retarded Green functions in real time are obtained by analytic continuation.

In the high-temperature limit, where all the momenta and masses of the internal particles are assumed to be much smaller than temperature, the effective action in fact turns out to be invariant under conformal rescalings, so (4) on a curved space with vanishing conformal Weyl tensor can be reconstructed by the simple transformation

$$
\begin{aligned}
& \left.\Pi_{\mu \nu \rho \sigma}(x, y)\right|_{g_{\mu \nu}=\sigma \eta_{\mu \nu}} \\
& \quad=\left.\sigma(x) \int \frac{d^{4} k}{(2 \pi)^{4}} e^{i k(x-y)} \tilde{\Pi}_{\mu \nu \rho \sigma}(k)\right|_{\eta} \sigma(y) .
\end{aligned}
$$

The Planck mass, which we assumed to be much larger than temperature, does not explicitly appear in $\tilde{\Pi}$ since we are treating the metric field as classical and no higher loop diagrams with graviton self-interactions are involved. So we only need to assume that the (zero-temperature) masses of the thermal matter are small compared to temperature (i.e., the plasma is ultrarelativistic), and that the relevant momentum scales are likewise so. Fortunately, this is just the case of interest with cosmological perturbations. If the latter have typical wavelengths of the order of the Hubble horizon, then $k / T \sim \sqrt{G T^{2}} \propto T / m_{\text {Planck }} \ll 1$.

One-loop diagrams correspond to collisionless thermal matter which has only gravitational interactions. The leading temperature contributions to (14) have been first calculated in [10] (see also Ref. [11]) and turn out to have a universal structure, where only the overall factor varies among the various forms of thermal matter according to their energy density. It is highly nonlocal and comes with a complicated tensor structure, since with $\eta^{\mu \nu}, u_{\mu}=\delta_{\mu}^{0}$, and $K^{\mu}=\left(K^{0}, \mathbf{k}\right)$, one can build 14 tensors to form a basis for $\tilde{\Pi}^{\mu \nu \alpha \beta}(K)=\rho \sum_{i=1}^{14} c_{i}(K) T_{i}^{\mu \nu \alpha \beta}(K)$, see Table $\mathbb{\text { q. }}$.

However, $\tilde{\Pi}$ satisfies the Ward identities corresponding to diffeomorphism invariance and conformal invariance, and this reduces the number of independent structure functions to 3. They can be chosen as

$$
A(K) \equiv \tilde{\Pi}_{0000}(K) / \rho, \quad B(K) \equiv \tilde{\Pi}_{0 \mu}{ }^{\mu}{ }_{0}(K) / \rho, \quad C(K) \equiv \tilde{\Pi}_{\mu \nu}{ }^{\mu \nu}(K) / \rho
$$

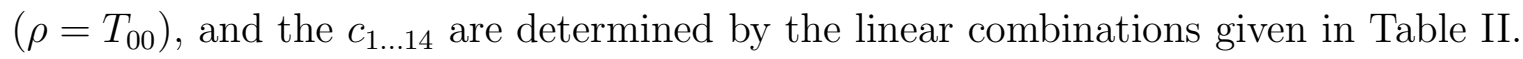

The universal result for ultrarelativistic collisionless thermal matter then reads

$$
A^{(1)}(K)=\omega \operatorname{artanh} \frac{1}{\omega}-\frac{5}{4}, \quad B^{(1)}=-1, \quad C^{(1)}=0,
$$

with $\omega \equiv K_{0} / k$.

Cosmological perturbations can be classified according to their transformation behaviour under spatial coordinate transformations [四] as scalar, vector, or tensor, which corresponds to compressional, rotational, or radiative perturbations in the plasma. The above 3 independent components of $\tilde{\Pi}$ determine, in certain combinations, the connection between the respective perturbations in the energy-momentum tensor and in the metric field. 
In the radiation-dominated epoch the standard choice is that of a spatially flat Einsteinde Sitter model with line element

$$
d s^{2}=\sigma(\tau)\left(d \tau^{2}-d \mathbf{x}^{2}\right), \quad \sigma(\tau)=\frac{8 \pi G \rho_{0}}{3} \tau^{2},
$$

( $\rho_{0}$ is the energy density when $\sigma=1$ ), and, given (8), it is moreover natural to decompose all perturbations in plane waves, since in linear perturbation theory the different modes evolve independently. The problem is thus reduced to a one-dimensional one, and it is convenient to introduce a dimensionless time variable

$$
x \equiv k \tau=\frac{R_{H}}{\lambda /(2 \pi)},
$$

which measures the (growing) size of the Hubble horizon over the wavelength of a given mode (which is constant in comoving coordinates).

Of all the numerous components of (1), only a few are independent by virtue of general covariance and turn out to involve only those gauge-invariant combinations of the components of the metric perturbations $\delta g_{\mu \nu}$ that have been studied by Bardeen [4]. For instance, the scalar part of metric perturbations can be parametrized in terms of four scalar functions

$$
\delta g_{\mu \nu}^{(S)}=\sigma(\tau)\left(\begin{array}{cc}
C & D_{, i} \\
D_{, j} & A \delta_{i j}+B_{, i j}
\end{array}\right)
$$

of which always two can be gauged away. Instead of fixing a gauge, we can also use the gauge-invariant combinations

$$
\begin{aligned}
& \Phi=A+\frac{\dot{\sigma}}{\sigma}\left(D-\frac{1}{2} \dot{B}\right) \\
& \Pi=\frac{1}{2}\left(\ddot{B}+\frac{\dot{\sigma}}{\sigma} \dot{B}+C-A\right)-\dot{D}-\frac{\dot{\sigma}}{\sigma} D,
\end{aligned}
$$

where a dot denotes differentiation with respect to the conformal time variable $\tau$.

Each spatial Fourier mode with wave vector $\mathbf{k}$ is related to perturbations in the energy density and anisotropic pressure according to

$$
\delta=\frac{1}{3} x^{2} \Phi, \quad \pi_{\text {anis. }}=\frac{1}{3} x^{2} \Pi .
$$

Here energy density perturbations $\delta$ are defined with respect to space-like hypersurfaces representing everywhere the local rest frame of the full energy-momentum tensor, whereas $\pi_{\text {anis. }}$ is an unambiguous quantity, since there is no anisotropic pressure in the background.

Correspondingly, when specifying to scalar perturbations, there are just two independent

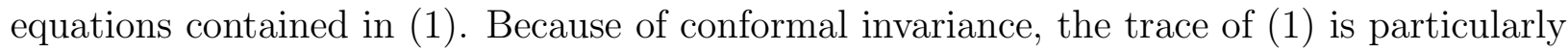
simple and yields a finite-order differential equation in $x$,

$$
\Phi^{\prime \prime}+\frac{4}{x} \Phi^{\prime}+\frac{1}{3} \Phi=\frac{2}{3} \Pi-\frac{2}{x} \Pi^{\prime}
$$

(a prime denotes differentiation with respect to the dimensionless time variable $x$ ). The other components, however, involve the nonlocalities of the gravitational polarization tensor. 
These lead to an integro-differential equation, which upon imposing retarded boundary conditions reads [8]

$$
\left(x^{2}-3\right) \Phi+3 x \Phi^{\prime}=6 \Pi-12 \int_{x_{0}}^{x} d x^{\prime} j_{0}\left(x-x^{\prime}\right)\left(\Phi^{\prime}\left(x^{\prime}\right)+\Pi^{\prime}\left(x^{\prime}\right)\right)+\varphi\left(x-x_{0}\right)
$$

where $j_{0}(x)=\sin (x) / x$ arises as Fourier transform of $A(\omega)$ in (7). $\varphi\left(x-x_{0}\right)$ encodes the initial conditions, the simplest choice of which corresponds to $\varphi\left(x-x_{0}\right) \propto j_{0}\left(x-x_{0}\right)$.

Similar integro-differential equations have been obtained from coupled Einstein-Vlasov equations in particular gauges, and the above one can be shown to arise from a gaugeinvariant reformulation of classical kinetic theory [12]. Usually, these equations were studied numerically, but in fact they can be solved analytically [8]. If initial conditions are formulated for $x_{0} \rightarrow 0$, a power series ansatz for $\Phi$ and $\Pi$ leads to recursion relations that can be solved and lead to an alternating series that converges faster than trigonometric functions.

This also holds true for the vector and tensor perturbations and when the more realistic case of a two-component system of perfect radiation fluid and ultrarelativistic plasma is considered [13].

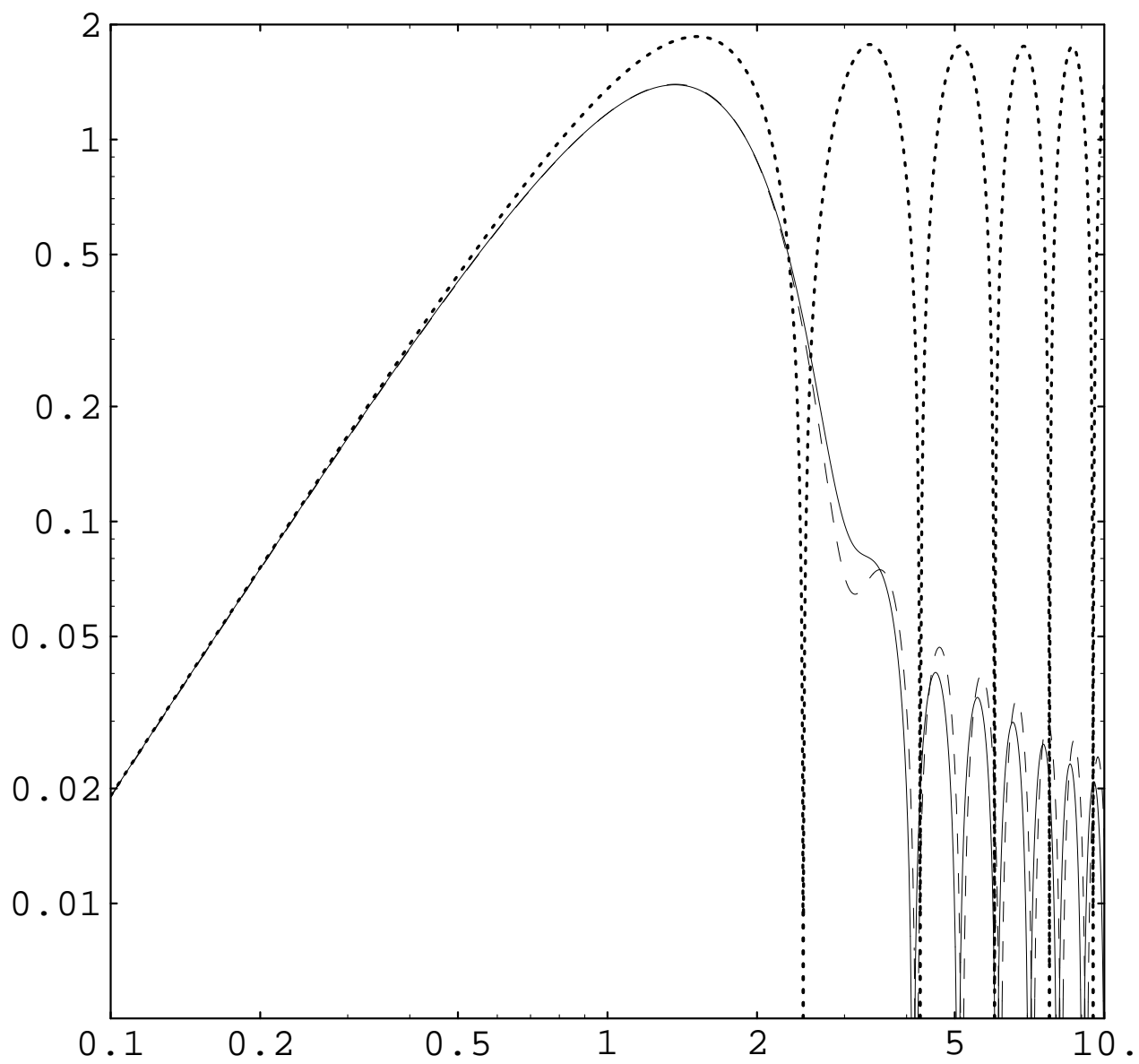

FIG. 1. The energy-density contrast (arbitrary normalization) as a function of $x / \pi$ for a collisionless ultrarelativistic plasma (full line), a scalar plasma with quartic self-interactions $\lambda \phi^{4}$ and $\lambda=1$ (dashed line), and a perfect radiation fluid (dotted line). 
In Fig. 1, the solution for the energy-density contrast is given in a doubly-logarithmic plot (full line) and compared with the perfect-fluid case (dotted line). In the latter, one has growth of the energy-density contrast as long as the wavelength of the perturbation exceeds the size of the Hubble radius $(x \ll 1)$. After the Hubble horizon has grown such as to encompass about one half wavelength $(x=\pi)$, further growth of the perturbation is stopped by the strong radiation pressure, turning it into an (undamped) acoustic wave propagating with the speed of sound in radiation, $v=1 / \sqrt{3}$. The collisionless case is similar as concerns the superhorizon-sized perturbations, but after horizon crossing, there is strong damping $\sim 1 / x$, and the phase velocity is about 1 . This indeed reproduces the findings of the numerical studies of Ref. [14]. They can be understood as follows: a energydensity perturbation consisting of collisionless particles propagates with the speed of their constituents, which in the ultrarelativistic case is the speed of light, and there is collisionless damping in the form of directional dispersion.

While with purely collisionless ultrarelativistic matter, all results are equivalent [12] to solving the classical Einstein-Vlasov equations, a quantum-field-theoretical treatment comes into its own when self-interactions within the thermal matter are taken into account. In a kinetic treatment one could add in a collision term to the coupled Einstein-Boltzmann equations, but eventually one would have to abandon the classical concept of a distribution function for the thermal matter. A virtue of the above thermal-field-theoretical approach is that everything is formulated in purely geometrical terms, without explicit recourse to perturbations in the (gauge variant) distribution function.

In Ref. [15], the gravitational polarization tensor has been calculated in a $\lambda \phi^{4}$ theory through order $\lambda^{3 / 2}$. The next-to-leading order contributions to $\Pi_{\mu \nu \alpha \beta}$ at order $\lambda^{1}$ are contained in the high-temperature limit of two-loop diagrams and their evaluation is straightforward. However, starting at three-loop order, there are infrared divergences which signal a breakdown of the convential perturbative series. This is caused by the generation of a thermal mass $\propto \sqrt{\lambda} T$ for the hot scalars. If this is not resummed into a correspondingly massive scalar propagator, repeated insertions of scalar self-energy diagrams in a scalar line produces arbitrarily high powers of massless scalar propagators all with the same momentum, and thus increasingly singular infrared behaviour (Fig. 2a).

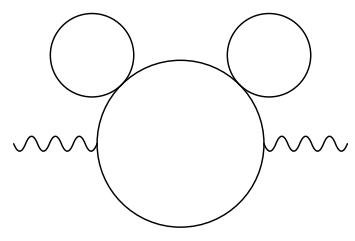

(a)

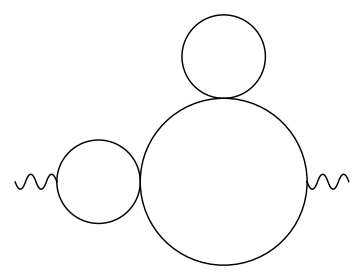

(b)

FIG. 2. Two examples of infrared divergent graphs beyond two-loop order.

However, it is not sufficient to resum this thermal mass for the hot scalars. After all, this would break conformal invariance. Indeed, there are also vertex subdiagrams $\propto \lambda T^{2}$ that have a similar effect as a self-energy insertion, see. Fig. 2 $2 \mathrm{~b}$. As in the hard-thermal-loop resummation program developed for high-temperature quantum chromodynamics [16], one has to resum also nonlocal vertex contributions. Doing so, the result turns out to satisfy 
both the diffeomorphism and conformal Ward identities.

In the low-momentum limit that is of interest in our application to cosmological perturbations, the function $A$ in (7) that governs the evolution of scalar perturbations reads through order $\lambda^{3 / 2}$

$$
\begin{aligned}
A= & \omega \operatorname{artanh} \frac{1}{\omega}-\frac{5}{4} \\
+ & \frac{5 \lambda}{8 \pi^{2}}\left[2\left(\omega \operatorname{artanh} \frac{1}{\omega}\right)^{2}-\omega \operatorname{artanh} \frac{1}{\omega}-\frac{\omega^{2}}{\omega^{2}-1}\right] \\
+ & \frac{5 \lambda^{3 / 2}}{8 \pi^{3}}\left[3\left(\omega^{2}-1-\omega \sqrt{\omega^{2}-1}\right)\left(\omega \operatorname{artanh} \frac{1}{\omega}\right)^{2}\right. \\
& +6\left(\omega \sqrt{\omega^{2}-1}-\omega^{2}-\frac{\omega}{\sqrt{\omega^{2}-1}}\right) \omega \operatorname{artanh} \frac{1}{\omega} \\
& \left.+\frac{\omega}{\left(\omega^{2}-1\right)^{3 / 2}}+3 \frac{\omega^{2}}{\omega^{2}-1}+6 \frac{\omega}{\sqrt{\omega^{2}-1}}-3 \omega \sqrt{\omega^{2}-1}+3 \omega^{2}\right]
\end{aligned}
$$

and similarly complicated expressions arise for $B$ and $C$, which in the collisionless limit were pure numbers.

The Fourier transform of this expression determines the kernel in the convolution integral of (15). At order $\lambda^{1}$, it can still be expressed in terms of well-known special functions [9], whereas at order $\lambda^{3 / 2}$ this would involve rather intractable integrals over Lommel functions. However, all that is needed for finding analytical solutions is their power series representations which are comparatively simple. Given them, it is as easy as before to solve the perturbation equations, however one finds that the asymptotic behaviour $x \gg 1$ eventually becomes sensitive to higher and higher loop orders. The reason for this is that higher loop orders come with increasingly singular contributions at $\omega= \pm 1$ to $A(\omega)$, and the large- $x$ behaviour is dominated by the latter. This could be cured by a further resummation similar to the one introduced for hot quantum chromodynamics in Ref. [17], but it turns out that a particular Padé-approximant based on the perturbative result reflects the effects of this further resummation quite well [18]. The result for the density perturbations in a scalar plasma with $\lambda \phi^{4}$-interactions and $\lambda=1$ are shown in Fig. 1 by the dashed line, where it is compared with the collisionless case (full line) and the one of a perfect radiation fluid (dotted line). The effects of the self-interactions within the ultrarelativistic plasma become important only for $x \gtrsim \pi$, where the strong collisionless damping is somewhat reduced and the phase velocity is smaller than 1 .

A full analysis of scalar, vector, and tensor perturbations in the general case of a twocomponent system containing also a perfect radiation fluid is given in Ref. [18]. Let us just mention one of the more spectacular results, which arise in the case of vector (rotational) perturbations. This case has not been investigated much previously, presumably because in the perfect-fluid case there are no regular solutions — rotational perturbations necessarily lead to strongly anisotropic initial singularities. This can be explained by the HelmholtzKelvin circulation theorem [19] which states that in a perfect fluid the circulation around a closed curve following the motion of matter is conserved. However, this theorem does not apply generally. Indeed, in a (nearly) collisionless medium one can have small initial anisotropies in the distribution function that gives rise to a growing vorticity on superhorizon 
scales [13, which decays after horizon crossing through directional dispersion. In a twocomponent system one can even have such perturbations which do not decay by arranging for vorticity in a perfect-fluid component that is compensated by an initially matching one with reversed sign in the nearly collisionless plasma component. Then net vorticity is generated by the decay of the vector perturbation in the plasma component, see Fig. 3. This is particularly interesting in that vector perturbations generally lead to the generation of primordial magnetic fields at the time when the universe changes from radiation to matter domination [20]. Because tiny primordial magnetic fields can act as seed fields for galactic dynamos, such rotational perturbations may therefore be of interest with respect to the still unsolved problem of the origin of galactic and intergalactic magnetic fields.

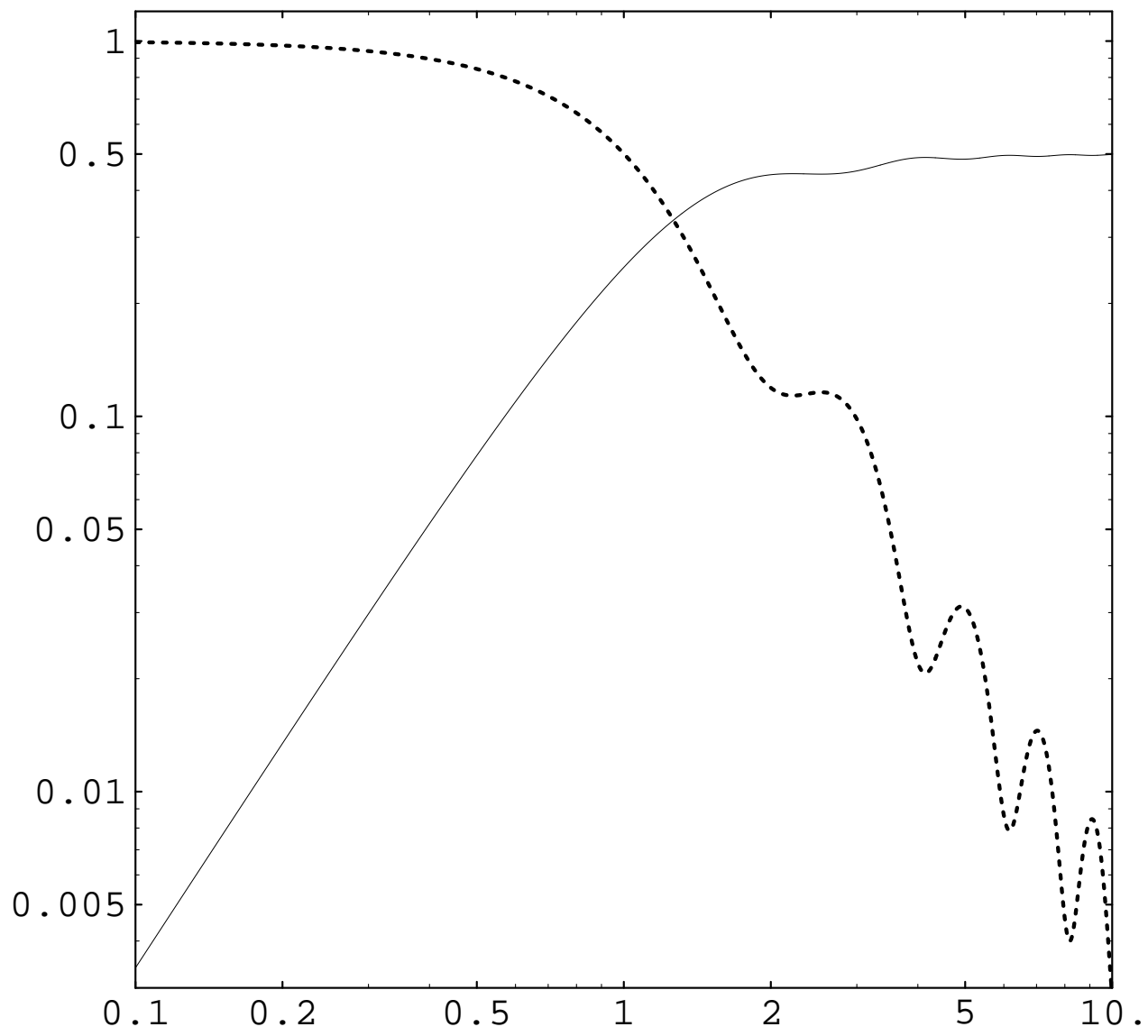

FIG. 3. Rotational perturbations in a two-component system consisting of $50 \%$ perfect radiation fluid and $50 \%$ ultrarelativistic scalar plasma $(\lambda=1)$. Given is the velocity amplitude for the total system (full line) and the weakly interacting plasma (dashed line) in arbitary units as a function of $x / \pi$.

\section{ACKNOWLEDGMENTS}

I am grateful to Ulli Kraemmer, Herbert Nachbagauer, and Dominik Schwarz, for their most enjoyable collaboration in various stages of the presented work. 


\section{TABLES}

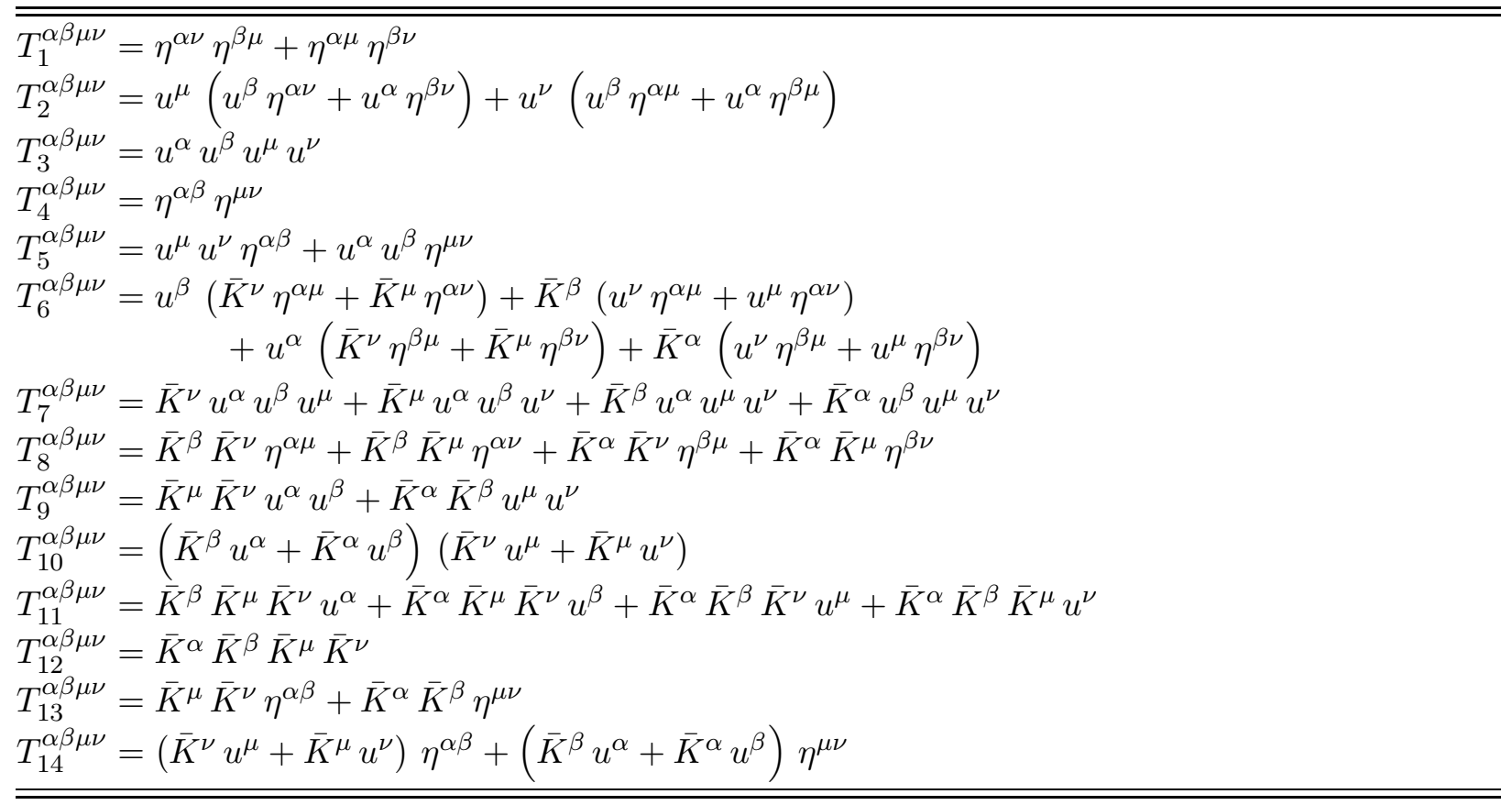

TABLE I. A basis of 14 independent tensors $T_{i}^{\alpha \beta \mu \nu}$ built from $\eta^{\mu \nu}, u^{\mu}=\delta_{0}^{\mu}$, and $\bar{K}^{\mu} \equiv K^{\mu} / k=(\omega, \mathbf{k} / k)$.

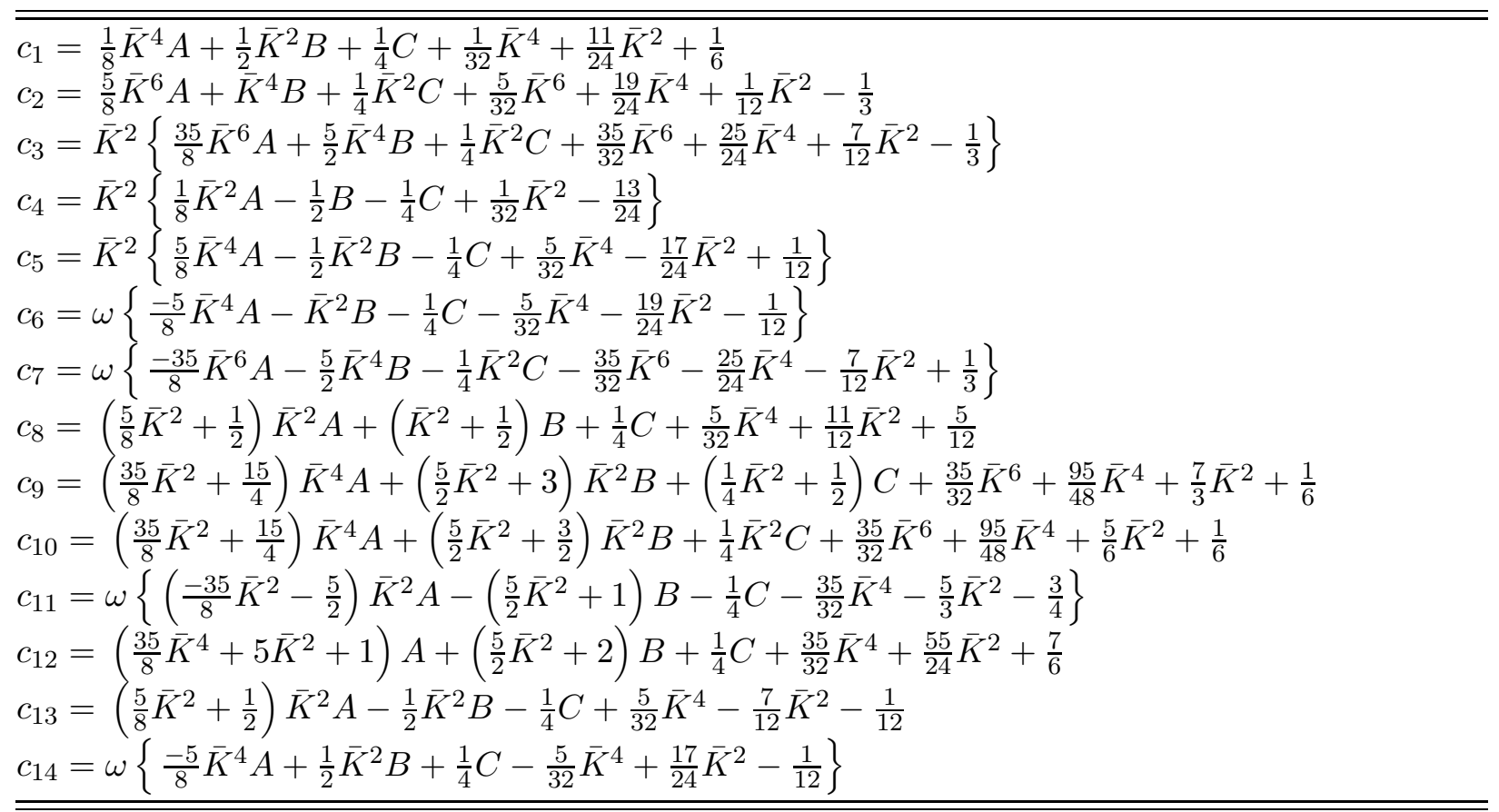

TABLE II. The structure of the conformally covariant gravitational polarization tensor $\tilde{\Pi}^{\mu \nu \alpha \beta} / \rho=\sum_{i=1}^{14} c_{i} T_{i}^{\mu \nu \alpha \beta}$ in terms of $A \equiv \tilde{\Pi}_{0000} / \rho, B \equiv \tilde{\Pi}_{0 \mu}{ }^{\mu}{ }_{0} / \rho$, and $C \equiv \tilde{\Pi}_{\mu \nu}{ }^{\mu \nu} / \rho$. 


\section{REFERENCES}

[1] G. Börner, The Early Universe: Facts and Fiction (Springer-Verlag, Berlin, 1993).

[2] G. F. Smoot et al., Astrophys. J. 396, L1 (1992).

[3] E. Lifshitz, Zh. Eksp. Teor. Fiz. 16, 587 (1946);

E. Lifshitz and I. Khalatnikov, Adv. Phys. 12, 185 (1963).

[4] J. M. Bardeen, Phys. Rev. D 22, 1882 (1980).

[5] G. F. R. Ellis and M. Bruni, Phys. Rev. D 40, 1804 (1989);

G. F. R. Ellis, J. Hwang and M. Bruni, Phys. Rev. D 40, 1819 (1989).

[6] J. Ehlers, in General Relativity and Gravitation, edited by R. K. Sachs (Academic Press, New York, 1971);

J. M. Stewart, Non-equilibrium Relativistic Kinetic Theory, (Springer-Verlag, New York, 1971).

[7] J. I. Kapusta, Finite-temperature field theory, (Cambridge University Press, Cambridge, 1989);

M. Le Bellac, Thermal Field Theory to appear in Cambridge University Press.

[8] U. Kraemmer and A. Rebhan, Phys. Rev. Lett. 67, 793 (1991).

[9] H. Nachbagauer, A. K. Rebhan, and D. J. Schwarz, Phys. Rev. D 51, R2504 (1995).

[10] A. Rebhan, Nucl. Phys. B351, 706 (1991).

[11] J. Frenkel and J. C. Taylor, Z. Phys. C 49, 515 (1991);

F. T. Brandt, J. Frenkel and J. C. Taylor, Nucl. Phys. B374, 169 (1992);

A. P. de Almeida, F. T. Brandt and J. Frenkel, Phys. Rev. D 49, 4196 (1994);

J. Frenkel, E. A. Gaffney and J. C. Taylor, Nucl. Phys. B439, 131 (1995).

[12] A. K. Rebhan and D. J. Schwarz, Phys. Rev. D 50, 2541 (1994).

[13] A. Rebhan, Nucl. Phys. B368, 479 (1992).

[14] J. R. Bond and A. S. Szalay, Astrophys. J. 274, 443 (1983).

[15] H. Nachbagauer, A. K. Rebhan, and D. J. Schwarz, preprint DESY 95-136, to appear in Phys. Rev. D (1995).

[16] E. Braaten and R. D. Pisarski, Nucl. Phys. B337, 569 (1990).

[17] U. Kraemmer, A. K. Rebhan and H. Schulz, Ann. Phys. (NY) bf 238, 286 (1995);

F. Flechsig and A. K. Rebhan, preprint DESY 95-170.

[18] H. Nachbagauer, A. K. Rebhan, and D. J. Schwarz, preprint DESY 95-191.

[19] B. J. T. Jones, Rev. Mod. Phys. 48, 107 (1976).

[20] A. Rebhan, Astrophys. J. 392, 385 (1992). 